\title{
Non-singlet coefficient functions for charged-current deep-inelastic scattering to the third order in QCD
}

\author{
J. Davies* \\ Department of Mathematical Sciences, University of Liverpool, Liverpool L69 3BX, UK \\ E-mail: Joshua.Davies@liv.ac.uk

\section{S. Moch} \\ II. Institute for Theoretical Physics, University of Hamburg, D-22761 Hamburg, Germany \\ E-mail: Sven-Olaf.Moch@desy.de
}

\section{J.A.M. Vermaseren}

Nikhef Theory Group, Science Park 105, 1098 XG Amsterdam, The Netherlands

E-mail: t68@nikhef.nl

\section{A. Vogt}

Department of Mathematical Sciences, University of Liverpool, Liverpool L69 3BX, UK

E-mail: Andreas.Vogteliv.ac.uk

\begin{abstract}
We have calculated the coefficient functions for the structure functions $F_{2}, F_{L}$ and $F_{3}$ in $v-\bar{v}$ charged-current deep-inelastic scattering (DIS) at the third order in the strong coupling $\alpha_{\mathrm{s}}$, thus completing the description of unpolarized inclusive $W^{ \pm}$-exchange DIS to this order of massless perturbative QCD. In this brief note, our new results are presented in terms of compact approximate expressions that are sufficiently accurate for phenomenological analyses. For the benefit of such analyses we also collect, in a unified notation, the corresponding lower-order contributions and the flavour non-singlet coefficient functions for $v+\bar{v}$ charged-current DIS. The behaviour of all six third-order coefficient functions at small Bjorken- $x$ is briefly discussed.
\end{abstract}

XXIV International Workshop on Deep-Inelastic Scattering and Related Subjects 11-15 April 2016, DESY Hamburg, Germany

\footnotetext{
* Speaker.
} 


\section{Introduction}

With six structure functions, $F_{2}, F_{L}$ and $F_{3}$ for $W^{+}$and $W^{-}$exchange [1,2], inclusive chargedcurrent DIS is an important source of information on the parton structure of nucleons and nuclei and on Standard Model parameters such as the strong coupling $\alpha_{\mathrm{s}}$ and the weak mixing $\sin ^{2} \theta_{W}$ [3]. Future facilities, e.g., the $\mathrm{LHeC}$ [4], will be required to fully realize its phenomenological potential. Charged-current structure functions are, at very small values of the Bjorken variable $x$, also of interest for the scattering of high-energy cosmic neutrinos, see Ref. [5] and references therein.

Here we address the corresponding coefficient functions (mass-factorized partonic cross sections) in massless perturbative QCD. These functions are relevant also beyond DIS, e.g., for Higgs production via vector boson fusion [6,7]. For recent progress on heavy-quark effects see Ref. [8].

\section{First-order and $v+\bar{v}$ non-singlet coefficient functions}

The first-order coefficient functions for unpolarized inclusive DIS were derived in the early days of QCD, see Refs. [1,9]. The second- and third-order contributions for the $v+\bar{v}$ charged-current case have been calculated in Refs. [10-17]. Here we collect the corresponding flavour non-singlet results in the $\overline{\mathrm{MS}}$ scheme for the standard choice $\mu_{F}=\mu_{R}=Q$ of the renormalization and factorization scales. The additional contributions at other values of $\mu_{F}$ and/or $\mu_{R}$ are determined by these results and the corresponding splitting functions [18,19]; see, e.g., Eqs. (2.16) - (2.18) in Ref. [20].

We denote the non-singlet quark coefficient functions for the charged-current structure functions $F_{2,3, L}^{v p \pm \bar{v} p}\left(x, Q^{2}\right)$ in neutrino-proton DIS by $C_{a, \pm}$, and write their perturbative expansion as

$$
C_{a, \pm}\left(x, Q^{2}\right)=\sum_{n=0} a_{\mathrm{s}}^{n} c_{a, \pm}^{(n)}(x) \quad \text { with } \quad a_{\mathrm{s}} \equiv \alpha_{\mathrm{s}}\left(Q^{2}\right) /(4 \pi) .
$$

In this notation the zeroth- and first-order coefficient functions are given by

$$
\begin{aligned}
& c_{2, \pm}^{(0)}(x)=c_{3, \pm}^{(0)}(x)=\delta\left(x_{1}\right), \quad c_{L, \pm}^{(0)}(x)=0, \quad c_{L, \pm}^{(1)}(x)=4 C_{F} x \\
& c_{2, \pm}^{(1)}(x)=C_{F}\left\{4 \mathscr{D}_{1}-3 \mathscr{D}_{0}-\left(9+4 \zeta_{2}\right) \delta\left(x_{1}\right)-2(1+x)\left(L_{1}-L_{0}\right)-4 x_{1}^{-1} L_{0}+6+4 x\right\} \\
& c_{3, \pm}^{(1)}(x)=c_{2, \pm}^{(1)}(x)-2 C_{F}(1+x)
\end{aligned}
$$

with $C_{F}=\left(n_{c}^{2}-1\right) /\left(2 n_{c}\right)=4 / 3$ in QCD. Here and below we use the abbreviations

$$
x_{1}=1-x, \quad L_{0}=\ln x, \quad L_{1}=\ln x_{1}, \quad \mathscr{D}_{k}=\left[x_{1}^{-1} L_{1}^{k}\right]_{+},
$$

where $[a(x)]_{+}$denotes +-distributions defined via $\int_{0}^{1} d x[a(x)]_{+} f(x) \equiv \int_{0}^{1} d x a(x)\{f(x)-f(1)\}$.

The coefficient functions $c_{a,+}^{(n)}$ and $c_{a,-}^{(n)}$ differ at $n>1$. The $2^{\text {nd }}$ - and $3^{\text {rd }}$-order contributions to the former quantities read, in an approximate but sufficiently accurate form given in Refs. [15-17],

$$
\begin{aligned}
c_{2,+}^{(2)}(x) \cong & 128 / 9 \mathscr{D}_{3}-184 / 3 \mathscr{D}_{2}-31.1052 \mathscr{D}_{1}+188.641 \mathscr{D}_{0}-338.513 \delta\left(x_{1}\right)-17.74 L_{1}^{3} \\
& +72.24 L_{1}^{2}-628.8 L_{1}-181.0-806.7 x+L_{0} L_{1}\left(37.75 L_{0}-147.1 L_{1}\right) \\
& +0.719 x L_{0}^{4}-28.384 L_{0}-20.70 L_{0}^{2}-80 / 27 L_{0}^{3} \\
+n_{f}\{ & 16 / 9 \mathscr{D}_{2}-232 / 27 \mathscr{D}_{1}+6.34888 \mathscr{D}_{0}+46.8531 \delta\left(x_{1}\right)-1.500 L_{1}^{2}+24.87 L_{1} \\
& \left.-7.8109-17.82 x-12.97 x^{2}+8.113 L_{0} L_{1}-0.185 x L_{0}^{3}+16 / 3 L_{0}+20 / 9 L_{0}^{2}\right\}, \\
c_{L,+}^{(2)}(x) \cong & -37.338+89.53 x+33.82 x^{2}+128 / 9 x L_{1}^{2}-46.50 x L_{1}+x L_{0}\left(32.90+18.41 L_{0}\right) \\
& -84.094 L_{0} L_{1}-128 / 9 L_{0}-0.012 \delta\left(x_{1}\right)+16 / 27 n_{f}\left\{6 x L_{1}-12 x L_{0}-25 x+6\right\},
\end{aligned}
$$




$$
\begin{aligned}
c_{3,+}^{(2)}(x) \cong & 128 / 9 \mathscr{D}_{3}-184 / 3 \mathscr{D}_{2}-31.1052 \mathscr{D}_{1}+188.641 \mathscr{D}_{0}-338.572 \delta\left(x_{1}\right)-16.40 L_{1}^{3} \\
& +78.46 L_{1}^{2}-470.6 L_{1}-149.75-693.2 x+0.218 x L_{0}^{4}+L_{0} L_{1}\left(33.62 L_{0}-117.8 L_{1}\right) \\
& -49.30 L_{0}-94 / 3 L_{0}^{2}-104 / 27 L_{0}^{3} \\
+n_{f} & \left\{16 / 9 \mathscr{D}_{2}-232 / 27 \mathscr{D}_{1}+6.34888 \mathscr{D}_{0}+46.8464 \delta\left(x_{1}\right)+0.066 L_{1}^{3}-0.663 L_{1}^{2}\right. \\
+ & \left.24.86 L_{1}-5.738-5.845 x-10.235 x^{2}-0.190 x L_{0}^{3}+4.265 L_{0} L_{1}+20 / 9 L_{0}\left(4+L_{0}\right)\right\}
\end{aligned}
$$

and

$$
\begin{aligned}
& c_{2,+}^{(3)}(x) \cong 512 / 27 \mathscr{D}_{5}-5440 / 27 \mathscr{D}_{4}+501.099 \mathscr{D}_{3}+1171.54 \mathscr{D}_{2}-7328.45 \mathscr{D}_{1}+4442.76 \mathscr{D}_{0} \\
& -9170.38 \delta\left(x_{1}\right)-512 / 27 L_{1}^{5}+704 / 3 L_{1}^{4}-3368 L_{1}^{3}-2978 L_{1}^{2}+18832 L_{1}-4926 \\
& +7725 x+57256 x^{2}+12898 x^{3}-56000 x_{1} L_{1}^{2}-L_{0} L_{1}\left(6158+1836 L_{0}\right)+4.719 x L_{0}^{5} \\
& -775.8 L_{0}-899.6 L_{0}^{2}-309.1 L_{0}^{3}-2932 / 81 L_{0}^{4}-32 / 27 L_{0}^{5} \\
& +n_{f}\left\{640 / 81 \mathscr{D}_{4}-6592 / 81 \mathscr{D}_{3}+220.573 \mathscr{D}_{2}+294.906 \mathscr{D}_{1}-729.359 \mathscr{D}_{0}\right. \\
& +2574.687 \delta\left(x_{1}\right)-640 / 81 L_{1}^{4}+153.5 L_{1}^{3}-828.7 L_{1}^{2}-501.1 L_{1}+831.6-6752 x \\
& -2778 x^{2}+171.0 x_{1} L_{1}^{4}+L_{0} L_{1}\left(4365+716.2 L_{0}-5983 L_{1}\right)+4.102 x L_{0}^{4}+275.6 L_{0} \\
& \left.+187.3 L_{0}^{2}+12224 / 243 L_{0}^{3}+728 / 243 L_{0}^{4}\right\} \\
& +n_{f}^{2}\left\{64 / 81 \mathscr{D}_{3}-464 / 81 \mathscr{D}_{2}+7.67505 \mathscr{D}_{1}+1.00830 \mathscr{D}_{0}-103.2366 \delta\left(x_{1}\right)-64 / 81 L_{1}^{3}\right. \\
& +18.21 L_{1}^{2}-19.09 L_{1}+129.2 x+102.5 x^{2}+L_{0} L_{1}\left(-96.07-12.46 L_{0}+85.88 L_{1}\right) \\
& \left.-8.042 L_{0}-1984 / 243 L_{0}^{2}-368 / 243 L_{0}^{3}\right\} \text {, } \\
& c_{L,+}^{(3)}(x) \cong 512 / 27 L_{1}^{4}-177.40 L_{1}^{3}+650.6 L_{1}^{2}-2729 L_{1}-2220.5-7884 x+4168 x^{2} \\
& -\left(844.7 L_{0}+517.3 L_{1}\right) L_{0} L_{1}+\left(195.6 L_{1}-125.3\right) x_{1} L_{1}^{3}+208.3 x L_{0}^{3}-1355.7 L_{0} \\
& -7456 / 27 L_{0}^{2}-1280 / 81 L_{0}^{3}+0.113 \delta\left(x_{1}\right) \\
& +n_{f}\left\{1024 / 81 L_{1}^{3}-112.35 L_{1}^{2}+344.1 L_{1}+408.4-9.345 x-919.3 x^{2}\right. \\
& +\left(239.7+20.63 L_{1}\right) x_{1} L_{1}^{2}+\left(887.3+294.5 L_{0}-59.14 L_{1}\right) L_{0} L_{1}-1792 / 81 x L_{0}^{3} \\
& \left.+200.73 L_{0}+64 / 3 L_{0}^{2}+0.006 \delta\left(x_{1}\right)\right\} \\
& +n_{f}^{2}\left\{3 x L_{1}^{2}+(6-25 x) L_{1}-19+\left(317 / 6-12 \zeta_{2}\right) x-6 x L_{0} L_{1}+6 x \operatorname{Li}_{2}(x)+9 x L_{0}^{2}\right. \\
& \left.-(6-50 x) L_{0}\right\} 64 / 81 \text {, } \\
& c_{3,+}^{(3)}(x) \cong 512 / 27 \mathscr{D}_{5}-5440 / 27 \mathscr{D}_{4}+501.099 \mathscr{D}_{3}+1171.54 \mathscr{D}_{2}-7328.45 \mathscr{D}_{1}+4442.76 \mathscr{D}_{0} \\
& -9172.68 \delta\left(x_{1}\right)-512 / 27 L_{1}^{5}+8896 / 27 L_{1}^{4}-1396 L_{1}^{3}+3990 L_{1}^{2}+14363 L_{1} \\
& -1853-5709 x+x x_{1}(5600-1432 x)-L_{0} L_{1}\left(4007+1312 L_{0}\right)-0.463 x L_{0}^{6} \\
& -293.3 L_{0}-1488 L_{0}^{2}-496.95 L_{0}^{3}-4036 / 81 L_{0}^{4}-536 / 405 L_{0}^{5} \\
& +n_{f}\left\{640 / 81 \mathscr{D}_{4}-6592 / 81 \mathscr{D}_{3}+220.573 \mathscr{D}_{2}+294.906 \mathscr{D}_{1}-729.359 \mathscr{D}_{0}\right. \\
& +2575.46 \delta\left(x_{1}\right)-640 / 81 L_{1}^{4}+32576 / 243 L_{1}^{3}-660.7 L_{1}^{2}+959.1 L_{1}+31.95 x_{1} L_{1}^{4} \\
& +516.1-465.2 x+x x_{1}(635.3+310.4 x)+L_{0} L_{1}\left(1496+270.1 L_{0}-1191 L_{1}\right) \\
& \left.-1.200 x L_{0}^{4}+366.9 L_{0}+305.32 L_{0}^{2}+48512 / 729 L_{0}^{3}+304 / 81 L_{0}^{4}\right\} \\
& +n_{f}^{2}\left\{64 / 81 \mathscr{D}_{3}-464 / 81 \mathscr{D}_{2}+7.67505 \mathscr{D}_{1}+1.00830 \mathscr{D}_{0}-103.2602 \delta\left(x_{1}\right)-64 / 81 L_{1}^{3}\right. \\
& +992 / 81 L_{1}^{2}-49.65 L_{1}+11.32-x x_{1}(44.52+11.05 x)+51.94 x+0.0647 x L_{0}^{4} \\
& \left.-L_{0} L_{1}\left(39.99+5.103 L_{0}-16.30 L_{1}\right)-16.00 L_{0}-2848 / 243 L_{0}^{2}-368 / 243 L_{0}^{3}\right\} \\
& +f l_{02} n_{f}\left\{2.147 L_{1}^{2}-24.57 L_{1}+48.79-x_{1}(242.4-150.7 x)-L_{0} L_{1}\left(81.70+9.412 L_{1}\right)\right. \\
& \left.+x L_{0}\left(218.1+82.27 L_{0}^{2}\right)-477.0 L_{0}-113.4 L_{0}^{2}+17.26 L_{0}^{3}-16 / 27 L_{0}^{5}\right\} x_{1} .
\end{aligned}
$$




\section{3. $v-\bar{v}$ non-singlet coefficient functions}

The differences between corresponding $v+\bar{v}$ and $v-\bar{v}$ coefficient functions are, as conjectured in Ref. [23], suppressed at large $x$ by two powers of $1-x$. Hence it is convenient to present the coefficient functions for $v-\bar{v}$ charged-current DIS in terms of differences which we define as

$$
\delta C_{2, L} \equiv C_{2, L}^{v p+\bar{v} p}-C_{2, L}^{v p-\bar{v} p}, \quad \delta C_{3} \equiv C_{3}^{v p-\bar{v} p}-C_{3}^{v p+\bar{v} p} .
$$

The flavour class $f l_{02}$, see Fig. 1 of Ref. [16], does not contribute to the flavour asymmetries probed in the $v-\bar{v}$ combinations, hence it is understood that the corresponding part of Eq. 2.11) is removed before the difference for $F_{3}$ is formed. The $\delta C_{a}$ can be perturbatively expanded as

$$
\delta C_{a}=\sum_{n=2} a_{\mathrm{s}}^{n} \delta c_{a}^{(n)}
$$

where $a_{\mathrm{s}}$ is defined in Eq. (2.1) above. The second-order results were already given in Ref. [21] in exact and parametrized form. The later results, written in terms of the abbreviations (2.5), read

$$
\begin{aligned}
\delta c_{2}^{(2)}(x) \cong\{ & -9.1587-57.70 x+72.29 x^{2}-5.689 x^{3}-x L_{0}\left(68.804+24.40 L_{0}\right. \\
& \left.\left.+2.958 L_{0}^{2}\right)+0.249 L_{0}+8 / 9 L_{0}^{2}\left(2+L_{0}\right)\right\} x_{1} \\
\delta c_{L}^{(2)}(x) \cong & \left\{10.663-5.248 x-7.500 x^{2}+0.823 x^{3}+x L_{0}\left(11.10+2.225 L_{0}\right.\right. \\
& \left.\left.-0.128 L_{0}^{2}\right)+64 / 9 L_{0}\right\} x_{1}^{2} \\
\delta c_{3}^{(2)}(x) \cong & \left\{-29.65+116.05 x-71.74 x^{2}-16.18 x^{3}+x L_{0}(14.60+69.90 x\right. \\
& \left.\left.-0.378 L_{0}^{2}\right)-8.560 L_{0}+8 / 9 L_{0}^{2}\left(4+L_{0}\right)\right\} x_{1} .
\end{aligned}
$$

The corresponding third-order corrections are the new results of the present contribution. They supersede the previous approximate expressions in Eqs. (3.7) - (3.9) of Ref. [21], which were based on the lowest five even- integer and odd-integer Mellin moments of $C_{3,-}$ and $C_{a,-}, a=2, L$, respectively, computed in Ref. [22]. Our new exact results can parametrized as

$$
\begin{aligned}
& \delta c_{2}^{(3)} \cong\left\{273.59-44.95 x-73.56 x^{2}+40.68 x^{3}+0.1356 L_{0}^{5}+8.483 L_{0}^{4}+55.90 L_{0}^{3}\right. \\
& +120.67 L_{0}^{2}+388.0 L_{0}-329.8 L_{0} L_{1}-x L_{0}\left(316.2+71.63 L_{0}\right)+46.30 L_{1} \\
& \left.+5.447 L_{1}^{2}\right\} x_{1}-0.0008 \delta\left(x_{1}\right) \\
& +n_{f}\left\{\left(-19.093+12.97 x+36.44 x^{2}-29.256 x^{3}-0.76 L_{0}^{4}-5.317 L_{0}^{3}-19.82 L_{0}^{2}\right.\right. \\
& \left.\left.-38.958 L_{0}-13.395 L_{0} L_{1}+x L_{0}\left(14.44+17.74 L_{0}\right)+1.395 L_{1}\right) x_{1}+0.0001 \delta\left(x_{1}\right)\right\}, \\
& \delta c_{L}^{(3)} \cong\left\{-620.53-394.5 x+1609 x^{2}-596.2 x^{3}+0.217 L_{0}^{3}+62.18 L_{0}^{2}+208.47 L_{0}\right. \\
& \left.-482.5 L_{0} L_{1}-x L_{0}\left(1751-197.5 L_{0}\right)+105.5 L_{1}+0.442 L_{1}^{2}\right\} x_{1}^{2} \\
& +n_{f}\left\{-6.500-12.435 x+23.66 x^{2}+0.914 x^{3}+0.015 L_{0}^{3}-6.627 L_{0}^{2}-31.91 L_{0}\right. \\
& \left.-x L_{0}\left(5.711+28.635 L_{0}\right)\right\} x_{1}^{2}, \\
& \delta c_{3}^{(3)} \cong\left\{-553.5+1412.5 x-990.3 x^{2}+361.1 x^{3}+0.1458 L_{0}^{5}+9.688 L_{0}^{4}+90.62 L_{0}^{3}\right. \\
& +83.684 L_{0}^{2}-602.32 L_{0}-382.5 L_{0} L_{1}-x L_{0}\left(2.805+325.92 L_{0}\right)+133.5 L_{1} \\
& \left.+10.135 L_{1}^{2}\right\} x_{1}-0.0029 \delta\left(x_{1}\right) \\
& +n_{f}\left\{\left(-16.777+77.78 x-24.81 x^{2}-28.89 x^{3}-0.7714 L_{0}^{4}-7.701 L_{0}^{3}-21.522 L_{0}^{2}\right.\right. \\
& \left.\left.-7.897 L_{0}-16.17 L_{0} L_{1}+x L_{0}\left(43.21+67.04 L_{0}\right)+1.519 L_{1}\right) x_{1}+0.00006 \delta\left(x_{1}\right)\right\} .
\end{aligned}
$$




\section{Discussion}

With the exception of the $n_{f}$ part of Eq. (2.7) and the $n_{f}^{2}$ part of Eq. (2.10) which are exact, the second- and third-order expressions in sections 2 and 3 have been obtained by fitting the coefficients not written as fractions in the non-distribution parts to the exact coefficient functions at $x \geq 10^{-6}$. Where useful, the coefficients of $\delta(1-x)$ have been adjusted (even from zero) to fine-tune the accuracy of Mellin moments and convolutions. The resulting accuracy of Eqs. 2.6 - 2.11) and (3.3) - (3.8) and their convolutions with typical quark distributions of hadrons is $0.1 \%$ or better except where the functions are very small. Towards smaller $x$ the accuracy deteriorates, but the results are still accurate to about $1 \%$ and $3 \%$ at $x=10^{-8}$ and $x=10^{-10}$, respectively.

FORTRAN subroutines of these functions can be obtained from the preprint server ARXIV.ORG by downloading the source of this note. They are also available from the authors upon request.

Analogous parametrizations for the pure-singlet quark and gluon coefficient functions for $F_{2}$ and $F_{L}$ have been given in Ref. [15] and section 4 of Ref. [16]. The partly very lengthy exact expression corresponding to Eqs. (2.6) - (2.11) and (3.3) - (3.5) can be found in Ref. [16] - where the $f l_{11}$ contribution has to be disregarded for the present charged-current case - and Refs. [17,21]; those for Eqs. (3.6) - (3.8) will be presented in Ref. [25]. Only the latter expressions allow for the analytical calculation of all integer Mellin moments of the coefficient functions.

The second moments of $\delta c_{2}^{(3)}$ and $\delta c_{L}^{(3)}$ are of particular relevance, since they enter the QCD corrections to the Paschos-Wolfenstein relation [24] for the determination of $\sin ^{2} \theta_{W}$ from chargedcurrent DIS [3]. The truncated numerical values of these moments for $n_{f}$ light flavours are

$$
\delta c_{2}^{(3)}(N=2)=-20.4001+0.72202 n_{f}, \quad \delta c_{L}^{(3)}(N=2)=-24.7755+0.80134 n_{f} .
$$

Within their error estimates, the previous approximate results for these moments [21] agree with Eq. (4.1). The corresponding analytical expressions will also by presented in Ref. [25].
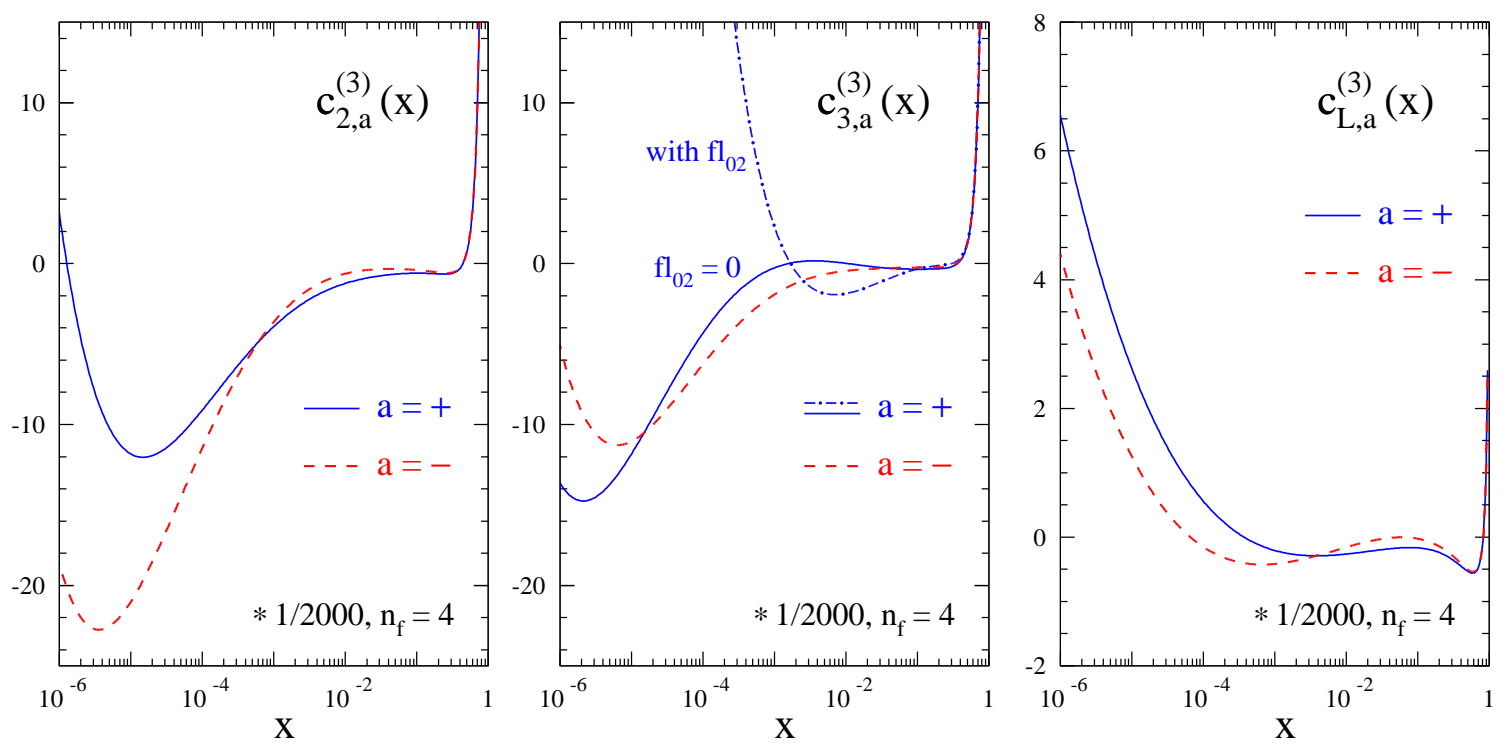

Figure 1: The third-order non-singlet coefficient functions for $F_{2}, F_{L}$ and $F_{3}$ in $v p+\bar{v} p(a=+)$ and $v p-\bar{v} p$ $(a=-)$ charged-current DIS for four light flavours. The function $c_{3,+}^{(3)}(x)$ in Eq. (2.11) is shown with and without the $f l_{02}$ contribution. The factor $1 / 2000$ approximately converts the curves to an expansion in $\alpha_{\mathrm{s}}$. 
The $a_{\mathrm{s}}^{3}$ coefficients $c_{a, \pm}^{(3)}$ in Eq. (2.1) are illustrated in Fig. 1 over a wide range in $x$. All six functions exhibit a sharp small- $x$ rise, but only at $x<10^{-5}$ for $F_{2}$ and $F_{3}$ and at $x \lesssim 10^{-4}$ for $F_{L}$. With the exception of the flavour structure $f l_{02}$ that occurs at three loops for the first time but dominates $c_{a,+}^{(3)}$ at small $x$, at least four of five $\ln ^{k} x$ small- $x$ terms are required for a good approximation for $c_{2, \pm}^{(3)}$ and $c_{3, \pm}^{(3)}$, and all three such terms for $c_{L, \pm}^{(3)}$, even below the $x$-range shown in the figure. Further discussions and illustrations of these coefficient functions will be presented in Ref. [25].

\section{Acknowledgements}

This work has been supported by the UK Science \& Technology Facilities Council (STFC) grants ST/L000431/1 and ST/K502145/1, by the Deutsche Forschungsgemeinschaft (DFG) via contract MO 1801/1-1, and by the European Research Council (ERC) Advanced Grant 320651, HEPGAME.

\section{References}

[1] A.J. Buras, Rev. Mod. Phys. 52 (1980) 199, and references therein

[2] K.A. Olive et al., Particle Data Group, Chin. Phys. C38 (2014) 090001

[3] G. Zeller et al., NuTeV, Phys. Rev. Lett. 88 (2002) 091802 E: ibid. 90 (2003) 239902, hep-ex/0110059

[4] J.L. Abelleira Fernandez et al., LHeC Study Group, J. Phys. G39 (2012) 075001, arXiv:1206.2913

[5] A.Y. Illarionov, B.A. Kniehl and A.V. Kotikov, Phys. Rev. Lett. 106 (2011) 231802, arXiv:1105.2829

[6] P. Bolzoni, F. Maltoni, S. Moch and M. Zaro, Phys. Rev. D85 (2012) 035002, arXiv:1109.3717

[7] F.A. Dreyer and A. Karlberg, arXiv:1606.00840

[8] J. Blümlein, G. Falcioni and A. De Freitas, arXiv:1605.05541

[9] W. Furmanski and R. Petronzio, Z. Phys. C11 (1982) 293, and references therein

[10] J. Sanchez Guillen et al., Nucl. Phys. B353 (1991) 337

[11] W.L. van Neerven and E.B. Zijlstra, Phys. Lett. B272 (1991) 127

[12] E.B. Zijlstra and W.L. van Neerven, Phys. Lett. B273 (1991) 476

[13] E.B. Zijlstra and W.L. van Neerven, Phys. Lett. B297 (1992) 377

[14] S. Moch and J.A.M. Vermaseren, Nucl. Phys. B573 (2000) 853, hep-ph/9912355

[15] S. Moch, J.A.M. Vermaseren and A. Vogt, Phys. Lett. B606 (2005) 123, hep-ph/0411112

[16] J.A.M. Vermaseren, A. Vogt and S. Moch, Nucl. Phys. B724 (2005) 3, hep-ph/0504242

[17] S. Moch, J.A.M. Vermaseren and A. Vogt, Nucl. Phys. B813 (2009) 220, arXiv:0812.4168

[18] S. Moch, J.A.M. Vermaseren and A. Vogt, Nucl. Phys. B688 (2004) 101, hep-ph/0403192

[19] A. Vogt, S. Moch and J.A.M. Vermaseren, Nucl. Phys. B691 (2004) 129, hep-ph/0404111

[20] W.L. van Neerven and A. Vogt, Nucl. Phys. B588 (2000) 345, hep-ph/0006154

[21] S. Moch, M. Rogal and A. Vogt, Nucl. Phys. B790 (2008) 317, arXiv:0708.3731

[22] S. Moch and M. Rogal, Nucl. Phys. B782 (2007) 51, arXiv:0704.1740

[23] D.J. Broadhurst, A.L. Kataev and C.J. Maxwell, Phys. Lett. B590 (2004) 76, hep-ph/0403037

[24] E.A. Paschos and L. Wolfenstein, Phys. Rev. D7 (1973) 91

[25] J. Davies, S. Moch, J.A.M. Vermaseren and A. Vogt, to appear 